\title{
A new automatic system for Cytomegalovirus antibodies screening: preliminary evaluation
}

\author{
Anna De Angelis, Francesca Dastoli, Maria De Cristofaro, Paola Spinozzi, Sabrina Pizziconi, \\ Alberto Spanò \\ U.O.C. Microbiologia, Virologia e Immunologia-Ospedale "Sandro Pertini"- ASL RMB - ROMA
}

Key words: Cytomegalovirus (CMV), Chemiluminescence, Serology

Valutazione di un sistema automatizzato per lo screening anticorpale dell'infezione da
Citomegalovirus

\section{SUMMARY}

The aim of our study was to evaluate the automated enhanced-chemiluminescence VITROS EciQ (Ortho) system compared to the EIA ALISEI (Radim) system for the first level detection of CMV IgG and IgM antibodies. Samples derived from 95 immunocompetent patients were examined. Both systems showed similar results for CMV IgG: 63 samples were positive, 28 negative and 4 samples were weakly positive. The four weakly positive samples were subsequently confirmed with the LIAISON (DiaSorin) system. Agreement was $91 \%$ concerning CMV IgM detection: 79 samples were negative, 7 positive and 9 samples releved discrepancy between the two systems. When these 9 cases were tested with LIAISON system for CMV IgM and IgG and the CMV IgG avidity test, data revealed complete agreement between VITROS EciQ and LIAISON systems. VITROS EciQ system seems to be a valuable system for the first level diagnosis of CMV infection.

\section{INTRODUZIONE}

Il Citomegalovirus umano (CMV) è uno degli otto virus erpetici che infettano la specie umana e, nel soggetto infettato, il virus risiede nelle feci, nel sangue, nell'urina e nelle secrezioni vaginali, orofaringee e cervicali (2).

Il virus si trasmette per contagio diretto e nella maggior parte dei casi, la malattia decorre asintomatica o con sintomi modesti, quali febbricola e astenia (2).

Una volta cessata la fase di attività, il virus assume una stabilità latente all'interno dell'organismo, pertanto in situazioni in cui le difese immunitarie siano compromesse, esso può riprendere la sua azione causando disturbi più importanti, come nel caso di trapianto d'organo $(4,7)$.

L'infezione da CMV nei pazienti sottoposti a trapianto costituisce un problema di grande rilevanza ed attualità; in questi pazienti, a causa dell'alterata risposta infiammatoria, l'espressività clinica appare frequentemente mascherata e la diagnosi è essenzialmente affidata ad indagini di laboratorio $(4,7)$.

Il CMV è la causa più comune di infezione virale congenita e perinatale (13).
Circa la metà delle donne gravide che acquisiscono il virus, trasmettono l'infezione al feto, con una probabilità di trasmissione tanto più alta quanto più è avanzata l'epoca di gestazione, anche se un'infezione contratta nel terzo trimestre causa danni al feto meno gravi di quelli causati da un'infezione contratta nel primo trimestre di gestazione (1).

Dal 10\% al 20\% dei neonati con infezione congenita, sviluppano sintomi acuti alla nascita e serie conseguenze cliniche.

Pertanto è fondamentale poter stabilire una corrispondenza tra un movimento sierologico, soprattutto a carico delle Ig di classe $\mathrm{M}$, e la reale presenza di una infezione insorta durante il periodo gravidico (5).

Quindi è fondamentale per il Laboratorio poter disporre di metodiche sensibili per evidenziare anche piccoli movimenti di IgM, ma altrettanto specifiche per evitare di sottoporre le pazienti ad approfondimenti diagnostici dispendiosi (3) e, spesso non del tutto privi di rischi $(6,12)$, o a terapie inutili oltre ad uno stress psicologico.

In considerazione dell'elevato numero di richieste presso il nostro Laboratorio del test di screening 
per CMV da parte di donne gravide o in previsione di una imminente gravidanza, abbiamo ritenuto interessante valutare un metodo in chemiluminescenza potenziata (VITROS EciQ - OrthoJohnson \& Johnson) per una diagnosi di primo livello, in alternativa al test immunoenzimatico (EIA) in micropiastra (ALISEI - Radim), attualmente utilizzato presso il nostro Servizio.

\section{MATERIALI E METODI}

\section{Campioni}

L'indagine è stata condotta utilizzando 95 campioni di siero appartenenti a 85 femmine e 10 maschi. Di tutti questi sieri sono state dosate le IgG e le IgM con entrambi i metodi. Sui campioni discordanti è stata effettuata una conferma con il sistema LIAISON (DiaSorin).

\section{VITROS CMV IgM e CMV IgG (Ortho- Johnson \& Johnson)}

I due dosaggi VITROS CMV IgM e VITROS CMV IgG, prodotti da Ortho-Johnson \& Johnson, recentemente commercializzati a completamento del pannello ToRC, sono stati eseguiti su VITROS EciQ, sistema random access, completamente automatico, in chemiluminescenza potenziata.

Il VITROS CMV IgG è un dosaggio immunometrico, che prevede la reazione degli anticorpi antiCMV presenti nel campione con antigene CMV adeso alla parete di un micropozzetto. Il coniugato, un anticorpo monoclonale di topo anti-IgG umane, marcato con HRP (perossidasi da rafano), permette la formazione di un complesso con l'anti-CMV legato alla parete del pozzetto. L'aggiunta di reagente, costituito da substrati luminogenici e agente di trasferimento elettroni, consente lo sviluppo di una reazione chemiluminescente. La HRP, legata alla parete del pozzetto, catalizza l'ossidazione del derivato del luminolo con sviluppo di luce; l'enhancer (acetanilide sostituita) incrementa il livello di luce prodotta e ne stabilizza l'emissione. Maggiore è la quantità di HRP adesa alla parete del pozzetto e maggiore sarà la quantità di luce emessa. Esiste quindi una proporzionalità diretta tra luce emessa e concentrazione di anti-CMV IgG.

Il VITROS CMV IgM è un dosaggio immunometrico a cattura, che prevede una prediluizione automatica del campione e reazione con anticorpo biotinilato monoclonale di topo anti-IgM umane. L'immunocomplesso viene catturato dalla streptoavidina, adesa alle pareti del pozzetto. Il coniugato, antigene CMV marcato con HRP, viene catturato dalle IgM CMV specifiche adese al pozzetto. L'aggiunta di reagente, costituito da substrati luminogenici e agente di trasferimento elettroni, consente lo sviluppo di una reazione chemilumi- nescente. La HRP, legata alla parete del pozzetto, catalizza l'ossidazione del derivato del luminolo con sviluppo di luce; l'enhancer (acetanilide sostituita) incrementa il livello di luce prodotta e ne stabilizza l'emissione. Maggiore è la quantità di HRP adesa alla parete del pozzetto e maggiore sarà la quantità di luce emessa. Esiste quindi una proporzionalità diretta tra luce emessa e concentrazione di anti-CMV IgM.

\section{ALISEI CMV IgG A CMV IgM EIA WELL (Radim)}

I dosaggi Citomegalovirus IgG ed IgM RADIM sono stati eseguiti su analizzatore automatico per micropiastre ALISEI.

Il dosaggio Citomegalovirus IgG è basato su metodo immunoenzimatico che utilizza quale marcatore la perossidasi. L'antigene CMV è adeso alle pareti del pozzetto e si lega ad eventuali anticorpi anti CMV IgG presenti nel campione. Il substrato tetrametilbenzidina (TMB) reagendo con la perossidasi, porta alla formazione di un complesso colorato, la cui intensità verrà misurata a 405 nm e $450 \mathrm{~nm}$. L'intensità di colore sarà direttamente proporzionale alla concentrazione degli anticorpi anti-CMV IgG presenti nel campione.

Il dosaggio Citomegalovirus IgM è basato su un principio EIA a cattura. Alla parete del pozzetto sono adesi anticorpi monoclonali anti catena $\mathrm{m}$ delle IgM umane. Il coniugato è rappresentato da una miscela di antigene CMV-anticorpo monoclonale anti-CMV biotinilato più streptoavidinaHRP. Il substrato è rappresentato da tetrametilbenzidina (TMB) incolore, che reagendo con la perossidasi, darà un composto colorato, la cui intensità verrà letta a $405 \mathrm{~nm}$ e $450 \mathrm{~nm}$.

\section{RANGE DI NORMALITÀ CMV IgM e IgG Ortho-Radim}

\begin{tabular}{llll}
\hline & Negativo & \multicolumn{2}{l}{ Limite/deb.pos Positivo } \\
\hline Ortho IgM & $<0.90 \mathrm{~s} / \mathrm{co}$ & $0.90-1.2 \mathrm{~s} / \mathrm{co}$ & $>1.2 \mathrm{~s} / \mathrm{co}$ \\
\hline Ortho IgG & $<4.99 \mathrm{U} / \mathrm{mL}$ & $5.0-7.99 \mathrm{U} / \mathrm{mL}$ & $>8.0 \mathrm{U} / \mathrm{mL}$ \\
\hline Radim IgM & $<0.9 \mathrm{Index}$ & $0.9-1.1 \mathrm{Index}$ & $>1.1 \mathrm{Index}$ \\
\hline Radim IgG & $<1.4 \mathrm{I} \mathrm{UI} / \mathrm{mL}$ & $1.41-4.2 \mathrm{UI} / \mathrm{mL}$ & $>4.2 \mathrm{UI} / \mathrm{mL}$ \\
\hline
\end{tabular}

\section{Controllo dei campioni discordanti}

I campioni discordanti sono stati testati utilizzando il sistema automatizzato in chemiluminescenza LIAISON (DiaSorin), usato come metodo di conferma presso il nostro Laboratorio.

Sono state determinate $\lg G$, lgM e Avidità per lgG.

\section{LIAISON CMV IgG e CMV IgM (DiaSorin)}

Il metodo per la determinazione quantitativa di IgG specifiche anti-human CMV è un test indiretto basato sul principio della chemiluminescenza. L'antigene CMV viene usato per rivestire le parti- 
celle magnetiche (fase solida) ed un anticorpo monoclonale di topo è legato ad un derivato dell'isoluminolo (coniugato anticorpo-isoluminolo). Durante la prima incubazione, gli anticorpi antiCMV presenti nel campione legano la fase solida. Durante la seconda incubazione, l'anticorpo coniugato reagisce con le IgG anti-CMV già legate alla fase solida. In seguito, vengono aggiunti i reagenti starter che inducono una reazione di chemiluminescenza. Il segnale luminoso, e quindi la quantità di coniugato anticorpo-isoluminolo, è misurato da un fotomoltiplicatore in unità relative di luce (RLU) ed è indicativo della concentrazione di IgG anti-CMV presente nel campione.

\section{RANGE DI NORMALITÀ CMV IgM e IgG DiaSorin}

\begin{tabular}{llll}
\hline & Negativo & Limite/deb.pos Positivo \\
\hline DiaSorin IgM & $<15 \mathrm{AU} / \mathrm{mL}$ & $15-30 \mathrm{AU} / \mathrm{mL}$ & $>30 \mathrm{AU} / \mathrm{mL}$ \\
\hline Diasorin IgG & $<0.4 \mathrm{UI} / \mathrm{Ml}$ & $0.4-0.6 \mathrm{Ul} / \mathrm{mL}$ & $>0.6 \mathrm{UI} / \mathrm{mL}$ \\
\hline
\end{tabular}

\section{LIAISON CMV IgG Avidità (DiaSorin)}

Il test di avidità è stato eseguito con la strumentazione LIAISON (11).

Il test di avidità delle IgG si basa sul principio secondo cui le IgG subiscono, durante l'evoluzione della risposta immunitaria, un aumento dell'affinità di legame per l'Antigene, che si traduce dal punto di vista diagnostico in un legame AntigeneAnticorpo meno sensibile all'azione di un agente denaturante quale l'urea. Ne deriva che più la produzione di IgG è temporalmente vicina, minore sarà la forza del legame all' antigene e minore sarà l'avidità; ciò permette di datare con buona attendibilità l'infezione.

Il test LIAISON CMV IgG Avidity impiega la tecnologia della chemiluminescenza (CLIA) in un saggio immunologico per la determinazione dell'avidità di legame di anticorpi specifici IgG diretti contro il CMV in campioni di siero o plasma umano.

Lo strumento calcola automaticamente l'indice di avidità di legame delle IgG specifiche antiCitomegalovirus (rapporto del campione trattato con urea rispetto al campione di riferimento) e classifica i risultati.

I risultati dei campioni devono essere interpretati come segue:

- un valore di indice di avidità al di sotto di 0.2 è da classificare avidità bassa;

- un valore di indice di avidità compreso tra $0.2 \mathrm{e}$ 0.3 è da classificare avidità moderata;

- un valore di indice di avidità uguale o al di sopra di 0.3 è da classificare avidità elevata.

\section{Controlli di qualità}

In ogni seduta analitica sono stati inseriti i con- trolli di qualità interni (home-made e della ditta Biorad) sia per le IgG che per le IgM.

\section{RISULTATI}

\section{Determinazione di IgG}

Dei 95 campioni testati, 63 sono risultati positivi e 28 sono risultati negativi sia con ALISEI sia con VITROS.

4 campioni sono risultati debolmente positivi per le IgG sia in VITROS che in ALISEI e confermati in LIAISON (tabella 1).

Le IgM di questi 4 campioni sono risultate negative con tutti e tre i metodi utilizzati (tabella 2).

Tabella I. CMV IgG

\begin{tabular}{llccc}
\hline & & & Ortho \\
& & NEG & DEB.POS & POS \\
\hline & NEG & 28 & 0 & 0 \\
\hline Radim & DEB.POS & 0 & 0 & 0 \\
\hline \multicolumn{2}{c}{ POS } & 0 & 4 & 63 \\
\hline
\end{tabular}

Tabella 2. CMV IgG debolmente positivi

\begin{tabular}{cccc}
\hline ALISEI IgG & VITROS IgG & LIAISON IgG & LIAISON IgM \\
\hline 8.52 & 7.54 & 2.8 & $<8$ \\
\hline 6.55 & 5.56 & 0.88 & 8.3 \\
\hline 7.42 & 7.35 & 1.2 & $<8$ \\
\hline 4.47 & 5.72 & 1.4 & $<8$ \\
\hline
\end{tabular}

\section{Determinazione di IgM}

Dei 95 campioni testati, 86 sono risultati concordanti per entrambi i metodi: 79 negativi e 7 positivi (tabella 3).

9 campioni sono risultati discrepanti (tabella 4):

- 4 campioni erano positivi in ALISEI, negativi in VITROS e confermati negativi in LIAISON; tali campioni sottoposti al test di avidità per CMV-IgG con il sistema LIAISON, sono risultati tutti con alta avidità, deponendo per una falsa positività dei risultati ottenuti con il sistema ALISEI;

- 1 campione era positivo in VITROS, negativo in ALISEI e confermato positivo in LIAISON; tale campione sottoposto al test di avidità per CMV-IgG con il sistema LIAISON, è risultato con moderata avidità, deponendo per una falsa negatività del risultato ottenuto con il sistema ALISEI;

- 3 campioni erano Grey-Zone in VITROS e negativi in ALISEI; di questi 2 campioni sono stati confermati con esito Grey-Zone in LIAISON e un campione è risultato negativo in LIAISON (pz n.9); tali campioni sottoposti al test di avidità per CMV-IgG con il sistema LIAISON, sono risultati tutti con alta avidità;

- 1 campione era Grey-Zone in ALISEI, negativo in VITROS e confermato negativo in LIAI- 
SON; tale campione sottoposto al test di avidità per CMV-IgG con il sistema LIAISON, è risultato con alta avidità, deponendo per una falsa debole positività del sistema ALISEI.

La figura I mostra la distribuzione di popolazione dei 95 campioni testati per CMV IgM, che, alla luce della valutazione dei dati ottenuti sui campioni discordanti, evidenzia una migliore specificità e sensibilità per il sistema VITROS EciQ, rispetto al sistema ALISEI.

Tabella 3. CMV IgM

\begin{tabular}{llccc}
\hline & NEG & $\begin{array}{c}\text { Ortho } \\
\text { LIMITE }\end{array}$ & POS \\
\hline & NEG & 79 & 3 & I \\
\hline Radim & LIMITE & I & 0 & 0 \\
\hline & POS & 4 & 0 & 7 \\
\hline
\end{tabular}

\section{DISCUSSIONE}

In relazione a tutte le problematiche concernenti una diagnosi sierologica da CMV, che può essere in alcune situazioni abbastanza semplice, ma in altre purtroppo molto complessa, il Laboratorio di Microbiologia assume un ruolo importante e delicato, per la qualità del dato, tant'è che alcune problematiche sono ancora in attesa di risoluzione, non ultima quella della scelta dei metodi e del flusso operativo da adottare, compresi l'organizzazione del lavoro e delle risorse umane.

Il sistema ALISEI in EIA utilizzato a tutt'oggi nel nostro Laboratorio, come primo screening per le IgG e IgM del CMV ha rappresentato un valido supporto diagnostico; d'altra parte gli avanzamenti tecnologici compiuti non solo dai Laboratori di ricerca, ma anche dalle ditte di diagnostici $(8,9)$, ci hanno indotto a valutare il suddetto sistema con quello VITROS in chemiluminescenza potenziata, in considerazione delle sue interessanti caratteristiche di controllo di integrità del risultato (Intellicheck), assenza totale di carry over garantita dall'uso di puntali monouso, facilità d'uso, elevata efficienza analitica, completo random access.

La buona concordanza con ALISEI per le IgG ha posto il sistema VITROS su un piano qualitativo

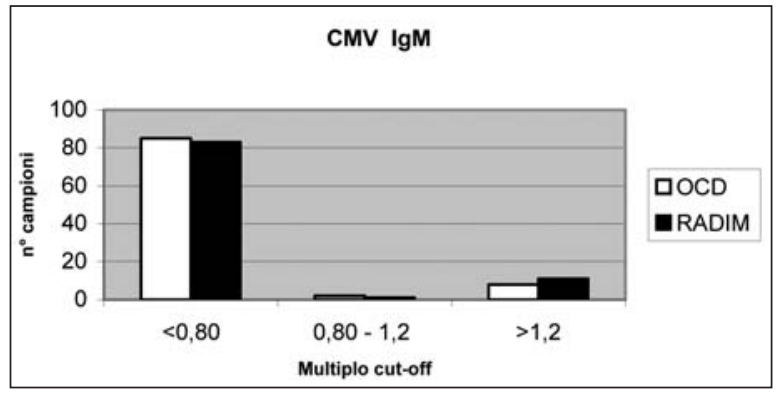

Figura I. Distribuzione di popolazione CMV IgM

di notevole interesse (tabella 1).

In letteratura è stato segnalato che alcuni test per IgG corrono il rischio di segnalare false positività, ma il sistema in valutazione VITROS non ha evidenziato, nella nostra esperienza, tale evenienza $(8,9,10)$.

Dai risultati ottenuti si evince che il sistema VITROS CMV IgG è perfettamente sovrapponibile sia per il metodo ALISEI che LIAISON (tabella 2).

La concordanza per CMV IgM tra i sistemi VITROS e ALISEI è risultata, in questo nostro studio preliminare, essere pari al 91\% (86/95) (tabella 3).

Per i pazienti con le IgM discordanti tra ALISEI e VITROS, è stata rilevata una ottima concordanza tra VITROS e LIAISON, deponendo per una probabile migliore sensibilità e specificità del metodo VITROS rispetto al metodo ALISEI (tabella 4).

Il test di avidità CMV IgG, utilizzato come test di approfondimento diagnostico per confermare o escludere la possibile infezione recente, applicato a tali campioni discordanti per le IgM tra ALISEI e VITROS, ha ulteriormente confermato la concordanza dei risultati tra i sistemi VITROS e LIAISON, basandosi solo sul dato analitico, purtroppo non supportato da notizie cliniche per mancanza di follow-up.

\section{CONCLUSIONI}

Il sistema VITROS si è dimostrato promettente per lo screening sierologico del CMV in una popolazione di soggetti immunocompetenti com-

Tabella 4. CMV IgM discrepanti (confronto tra ALISEI-VITROS-LIAISON)

\begin{tabular}{lccccc}
\hline PZ & ALISEI IgM & VITROS IgM & LIAISON IgM & LIAISON IgG & LIAISON IgG Avidity \\
\hline $\mathbf{I}$ & 1.55 & 0.7 & 10 (neg) & 5.3 & 0.423 (high) \\
\hline $\mathbf{2}$ & 0 & 0.93 & $26.6(\mathrm{G.Z})$. & 6.8 & 0.364 (high) \\
\hline $\mathbf{3}$ & 0.21 & 3.42 & $69(\mathrm{pos})$ & $>22$ & 0.277 (mod.) \\
\hline $\mathbf{4}$ & 0 & $\mathrm{I}$ & $26.4(\mathrm{G} . \mathrm{Z})$. & 1.9 & 0.572 (high) \\
\hline $\mathbf{5}$ & 1.13 & 0.79 & $<8(\mathrm{neg})$ & 20.2 & 0.484 (high) \\
\hline $\mathbf{6}$ & 1.25 & 0.69 & $<8(\mathrm{neg})$ & $>22$ & 0.615 (high) \\
\hline $\mathbf{7}$ & 0.94 & 0.65 & $<8(\mathrm{neg})$ & 3.7 & 0.701 (high) \\
\hline $\mathbf{8}$ & 1.66 & 0.74 & $<8(\mathrm{neg})$ & 7.5 & 0.508 (high) \\
\hline $\mathbf{9}$ & 0.35 & 1.05 & $<8(\mathrm{neg})$ & 5.4 & 0.539 (high) \\
\hline
\end{tabular}


prendenti prevalentemente donne in gravidanza, sia per la riproducibilità e la sicurezza del dato che per un risparmio economico, di tempi di refertazione e di carico di lavoro.

\section{BIBLIOGRAFIA}

1. Bodeus M, Hubinont C, Goubau P. Increased risk of Cytomegalovirus transmission in utero during late gestation. Obstet Gynecol 1999; 93: 658-60.

2. Britt WJ, Alford CA. Cytomegalovirus. In: Virology, Fields BN, Knipe DM, Howley PM Eds, Third Edition. Raven Press, New York 1996; 2493-523.

3. Eggers M, Metzger C, Enders G. Differentiation between acute primary and recurrent human Cytomegalovirus infection in pregnancy, using a microneutralization assay. J Med Virol 1998; 56: 351-8.

4. Fiala M, Payne JE, Berne TV, et al. Epidemiology of Cytomegalovirus infection after transplantation and immunosuppression. J Infect Dis 1975; 132 (4): 421-33.

5. Fowler KB, Stagno S, Pass RF, Britt WJ, Boll TJ, Alford CA. The outcome of congenital Cytomegalovirus infection in relation to maternal antibody status. $N$ Engl $J$ Med 1992; 326: 663-7.

6. Grose C, Meehan T, Weiner CP. Prenatal diagnosis of congenital Cytomegalovirus infection by virus isolation after amniocentesis. Pediatr Infect Dis J 1992; 11: 6057.

7. Ho M. Epidemiology of Cytomegalovirus infections. Rev Inf Dis 1990; 12: S701-10.

8. Lazzarotto T, Brojanac S, Maine GT, Landini MP. Search for Cytomegalovirus-specific Immunoglobulin M: comparison between a new Western-Blot, conventional Western-Blot and nine commercially available assays. Clin Diagn Lab Immunol 1997; 4: 483-6.

9. Lazzarotto T, Guerra B, Lanari M, Gabrielli L, Landini MP. Infezione congenita da Cytomegalovirus: possibilità significative della diagnostica di Laboratorio. Ligand Assay 2005; 10 (1): 9-14.

10. Priya K, Madhavan HN. Diagnostic value of enzyme linked immuno-sorbent assay for Cytomegalovirus disease. J PG Med 2002; 48: 176-8.

11. Revello MG, Gorini G, Gerna G. Clinical evaluation of a chemiluminescence immunoassay for determination of immunoglobulin G Avidity to human Cytomegalovirus. Clin Diagn Lab Immunol 2004; 11: 801-5.

12. Revello MG, Zavattoni M, Furione M, Baldanti F, Gerna G. Quantification of human Cytomegalovirus DNA in amniotic fluid of mothers of congenitally infected fetuses. J Clin Microbiol 1999; 37: 3350-2.

13. Stehel EK, Sanchez PJ. Cytomegalovirus infection in the fetus and neonate. NeoReviews 2005; 6: e38-e45. 